\title{
Role of serum CEA as tumor marker for predicting presence of pelvic and paraaortic lymph node metastasis in SCC of uterine cervix
}

\begin{abstract}
Objectives: Cancer of the uterine cervix is the major cause of death from gynecologic cancer worldwide. Isolated paraaortic lymph node metastasis detected at the initial diagnosis of cervical cancer could be addressed by extended field paraaortic lymph node irradiation. Serum Carcinoembryonic antigen (CEA) could be done for detecting early Para aortic lymph node (PALN) involvement and scrutinizing relapse or recurrence. To assess pelvic and paraaortic lymph node status by computed tomography scan and also correlate with the pretreatment serum Carcinoembryonic antigen levels.
\end{abstract}

Materials \&methods: Twenty one patients of histologically proven squamous cell carcinoma of cervix of any age group were recruited into the study. We assessed the status of pelvic and paraaortic lymph node by computed tomography scans study. Pretreatment serum CEA levels were evaluated in all patients irrespective of the stage from I to IV.

Results: We observed that there was an elevation of Carcinoembryonic antigen in those patients having paraaortic lymph node and PALN metastasis.

Conclusion: Based on the results, we could opine that serum Carcinoembryonic antigen levels could help to prognosticate the cervical cancer patient, predict the presence of paraaortic and pelvic lymph nodes. To explore the potential of for PALN detection and treatment response assessment.
Volume 8 Issue 2 - 2017

\author{
Chandana G,' Bhaskar Vishwanathan, ${ }^{2}$ \\ Mohamed Abdul Malik, ${ }^{3}$ Swathi Krishna \\ Reddy, ${ }^{4}$ Raghavendra DS ${ }^{5}$ \\ 'Department of Biochemistry, PES Institute of Medical Sciences \\ \& Research Centre, India \\ ${ }^{2}$ Department of Radiation oncology, Vydehi Institute of Medical \\ Sciences and research center, India \\ ${ }^{3}$ Department of Radiation oncology, Ashwin Hospital, India \\ ${ }^{4}$ Department of Genetics, Vydehi Institute of Medical Sciences \\ and research center, India \\ ${ }^{5}$ Department of Biochemistry, East Point Institute of Medical \\ Sciences and Research Centre, India
}

Correspondence: Swathi Krishna Reddy, Vydehi Institute of Medical Sciences and research center, \#82, EPIP area,Whitefield, Bangalore-560066, Karnataka, India, Tel 7204829222; Email 24swathi.reddy@gmail.com

Received: May 22, 2017 | Published: August 02, 2017

Keywords: cervical cancer, squamous cell carcinoma, carcino embryonic antigen, tumor marker

\section{Introduction}

Cancer of the uterine cervix is the major cause of death from gynecologic cancer worldwide. The reported incidence rates in developing countries are much higher than those in developed countries. Approximately $85 \%$ of cervical cancers are of the squamous cell type. Other histological types less frequently found include adenocarcinoma (approximately 10\%-15\%) and adenosquamous carcinoma (approximately $3 \%$ ). ${ }^{1}$ It is estimated that $50 \%$ of the women in whom cervical cancer is diagnosed each year will have never had cervical cytology testing. ${ }^{2}$ Abnormal vaginal bleeding, including postcoital, intermenstrual, and postmenopausal bleeding, is the most common symptom of cervical cancer. ${ }^{3}$ Studies of the incidence and distribution of lymph node metastasis in women with stage IB to IIB cervical cancer suggested that PALN involvement is closely tied to the presence of pelvic lymph node metastasis and larger primary tumour size. ${ }^{4}$

Analysis of outcome data from 555 women who participated in gynecologic oncology group (GOG) trials (GOG85, GOG120, GOG165) revealed a more positive prognosis for patients who underwent surgical exclusion of paraaortic lymph node involvement versus those who underwent radiographic determination of radiographic determination of paraaortic involvement. ${ }^{5}$

One study examined the efficacy of extending the radiation therapy (RT) field to the paraaortic region in patients with paraaortic lymph node involvement and showed therapeutic benefit especially in patients with small volume nodal disease. ${ }^{6}$ A randomized controlled trial examining surgical versus radiology staging and treatment of paraaortic lymph node involvement is ongoing. ${ }^{7}$. For patients suspicion of positive paraaortic and pelvic lymph node involvement imaging work up for metastatic disease is recommended. Extra peritoneal lymph node dissection should be considered followed by extended field external beam radiation therapy (EBRT), concurrent cisplatin containing chemotherapy and brachytherapy for patients with positive paraaortic lymph nodes. ${ }^{8}$

FIGO Staging for carcinoma cervix does not take into consideration the lymph node status of the patient. Para aortic was considered as distant metastasis in carcinoma cervix but at present considered as regional metastases. Lymphadenopathy cannot be judged clinically and it requires radiological investigations like $\mathrm{CT}$ scan and PET-CT Scan.

\section{Tumor markers}

A tumor marker is a substance that is produced by the body in response to cancer, or is produced by the cancer itself. Some of these markers are specific to one cancer, while others are seen in several types of cancer. These markers are generally used to evaluate the patient's response to treatment or to monitor for recurrence Tumor markers can be used in conjunction with other tests (scans, biopsies, etc.) to help diagnose a patient who has symptoms suspicious for cancer. Some markers can help physicians to determine prognosis and treatment.

Carcino embryonic antigen (CEA) is a glycoprotein of molecular weight approx. $180 \mathrm{KD}$ found in normal fetal gastrointestinal tissue; it is normally present at very low concentrations in adult plasma 
but its concentration is increased in the presence of many tumors, particularly colorectal cancers $(70 \%)$. Increased concentrations have also been described in gastric, bronchial, uterine and ovarian cancers, and in lymphomas.

Studies have focused on Carcinoembryonic antigen (CEA) for over 40 years, suggesting it be a useful tool in the diagnosis of cancer, as it is a cancer cell adhesion marker. Some of the nonneoplastic conditions associated with elevated CEA levels include cigarette smoking, peptic ulcer disease, inflammatory bowel disease, pancreatitis, hypothyroidism, biliary obstruction, and cirrhosis. Levels exceeding $10 \mathrm{ng}$ per $\mathrm{ml}$. are rarely due to benign disease. ${ }^{9}$

Elevated levels of CEA $(>2.5 \mathrm{ng} / \mathrm{ml})$ were first reported in patients with gynecologic malignancy by Lo Gerfo et al. ${ }^{10}$ Since that time, immunoelectrophoretic and column chromatographic studies have shown that CEA from cervical cancer and from colonic cancer are immunologically similar.

Carcinoembryonic antigen is a reliable tumor marker in patients with adenocarcinoma of the cervix. An excellent correlation has been noted between pre-treatment plasma levels and extent of the disease. High pre-treatment values were associated with bad prognosis. Contrary to squamous cell carcinomas, a low value is equivocal, meaning either that the tumor volume is small or that the tumor releases little or no CEA. The presence of lymph node metastases has more pronounced effect on the CEA values than the size of the primary tumor. ${ }^{11,12}$

Para-aortic lymphadenectomy or PET-CT examination is important for planning the treatment of radiotherapy. If isolated paraaortic lymph node metastasis is detected at the initial diagnosis of cervical cancer, the RT field should be extended to paraaortic lymph node lesions. To eliminate paraaortic lymph node micro metastasis and recurrence, more aggressive treatments such as Para-aortic lymphadenectomy, prophylactic paraaortic lymph node irradiation, or additional chemotherapy should be considered in future clinical trials. $^{13}$

Disaia et al. ${ }^{14}$ studied a group of patients with invasive squamous cell carcinoma of the cervix and found that there was a progressive increase in the percentage of patients with positive CEA values correlating with advancing stage of the disease from $26 \%$ in stage I to $88 \%$ in stage II. Incidentally $85 \%$ of the recurrent cases showed positive CEA values. ${ }^{14}$

Pre-treatment levels over 5ug/l are highly suggestive of metastatic disease as they are associated with metastases in pelvic or Paraaortic lymph nodes in $50 \%$ of patients with stage IB disease. Also in advanced stages such as III and IV, $48 \%$ of patients had a pre-therapy value exceeding 5 ug. ${ }^{15,16}$

\section{Methodology}

Twenty one Carcinoma cervix patients of any age group were examined prospectively who were admitted in the ward. They were clinically staged using FIGO staging classification. The procedure for staging included a detailed history and a physical examination, as well as common laboratory tests and standard chest radiographs, intravenous pyelograms, X-rays, cystoscopies, and sigmoidoscopies. In the evaluation of paraaortic and pelvic lymph node involvement, computed tomography (CT) was performed in all patients. The principle criterion for positive node involvement was based on the axial diameter of the lymph node. Lymph nodes larger than $1 \mathrm{~cm}$ in the short-axis dimension were considered abnormal. Then serum CEA levels were estimated by using two-site immunoenzymatic "sandwich" assay (BECKMAN COULTER ACCESS IMMUNOASSAY SYSTEM) in all patients irrespective of the stage from I to IV and nodal status before treatment. All these patients were considered based on the inclusion and exclusion criteria.

The Biopsy proven carcinoma of cervix patients from stage I to stage IV was included in the study. Metastasis to visceral organs and bones, Cigarette smokers, Confirmed or suspected cases of inflammatory bowel disorders such as ulcerative colitis and Chron's disease, pancreatitis, liver disease, pulmonary infections; secondary primary like colorectal, lung, gastric, breast, pancreatic, ovarian and uterine cancer, were excluded from the study.

\section{Statistical analysis}

Data analysis was performed with statistical package for social sciences (SPSS) version 21.0 statistical package. We have used MannWhitney $\mathrm{U}$ test for the analysis. The probability value $\mathrm{P}<0.05$ was considered as significant.

\section{Results and discussion}

The total of 21 patients of carcinoma cervix was taken up for the study.Age of patients ranged between 23-60years. Stage IB- 1 patient, Stage IIA -1patient, Stage IIB -6patient, Stage IIIB -6patient, Stage IVA -3patient and IVB -4patients were included in the study.

\section{Pelvic lymph nodes Vs CEA}

Most of the patients in the study were aged between $40-50 \mathrm{yrs}$. and majority of them were in advanced stages i.e. IIB, IIIB, IVB. Paraaortic lymph node was detected with CT scan in $60 \%$ of patients and $80 \%$ of patients there was pelvic lymph node involvement. CEA value $<3 \mathrm{ng} / \mathrm{ml}$ was taken as normal (Tables $1 \& 2$ ).

Table I Comparison of paraaortic lymph nodes present and paraaortic lymph nodes absent with respect to their CEA levels

\begin{tabular}{|c|c|c|c|c|c|}
\hline & \multicolumn{2}{|c|}{$\begin{array}{l}\text { Paraaortic lymph } \\
\text { node present }\end{array}$} & \multicolumn{2}{|c|}{$\begin{array}{l}\text { Paraaortic lymph } \\
\text { node absent }\end{array}$} & \multirow[t]{2}{*}{ P Value } \\
\hline & Median & $\begin{array}{l}\text { Interquartile } \\
\text { Range }\end{array}$ & Median & $\begin{array}{l}\text { Interqua } \\
\text { Range }\end{array}$ & \\
\hline CEA & 5.88 & 7.27 & 1.94 & 1.74 & 0.003 \\
\hline
\end{tabular}

Table 2 Comparison of pelvic lymph nodes present and pelvic lymph nodes absent with respect to their CEA levels

\begin{tabular}{llllll}
\hline & $\begin{array}{l}\text { Paraaortic lymph } \\
\text { node present }\end{array}$ & \multicolumn{2}{l}{$\begin{array}{l}\text { Pelvic lymph node } \\
\text { absent }\end{array}$} & P Value \\
\hline Median & $\begin{array}{l}\text { Interquartile } \\
\text { Range }\end{array}$ & Median & $\begin{array}{l}\text { Interquartile } \\
\text { Range }\end{array}$ & \\
CEA & 4.030 & 7.08 & 1.28 & 0.50 & 0.004 \\
\hline
\end{tabular}

Totally 21 patients participated in the study. Out of these 8 patients were paraaortic lymph nodes positive. All the $8(100 \%)$ patients showed their CEA $>3 \mathrm{ng} / \mathrm{ml} .15$ patients of the total 21 patients had their pelvic lymph nodes positive and $11(73.3 \%)$ patients among them had CEA $>3 \mathrm{ng} / \mathrm{ml}$.

We noted that CEA values were elevated in $73.3 \%$ of the patients who had pelvic lymph involvement. CEA values were also elevated more than the normal values in all the patients who had paraaortic lymph nodes involvement detected by CT scan, showing it to be an excellent tumor marker for paraaortic lymph node involvement (i.e. in $100 \%$ ) (Figures $1 \& 2$ ). 


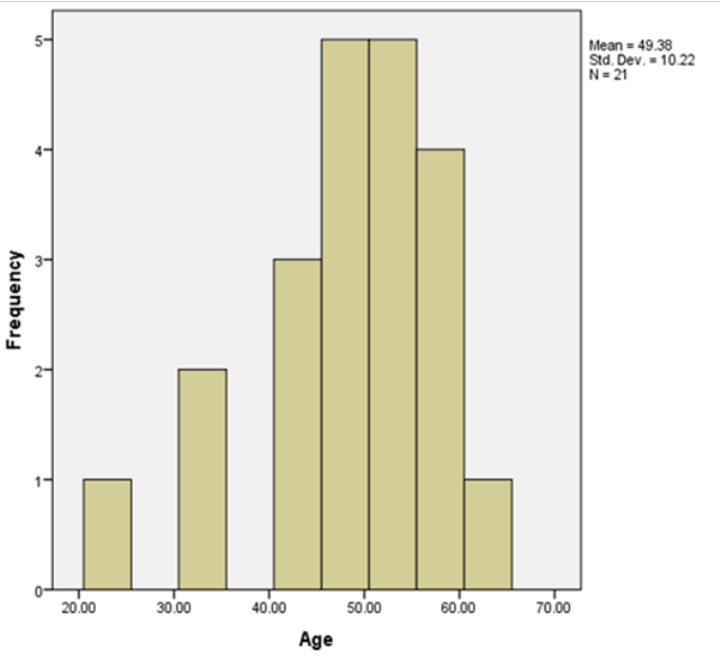

Figure I Histogram representing the age distribution of 21 participants. The minimum and maximum age of the participants who participated in the study was found to be 23,65 respectively. The mean + SD of the age distribution is $49.38+10.21$.

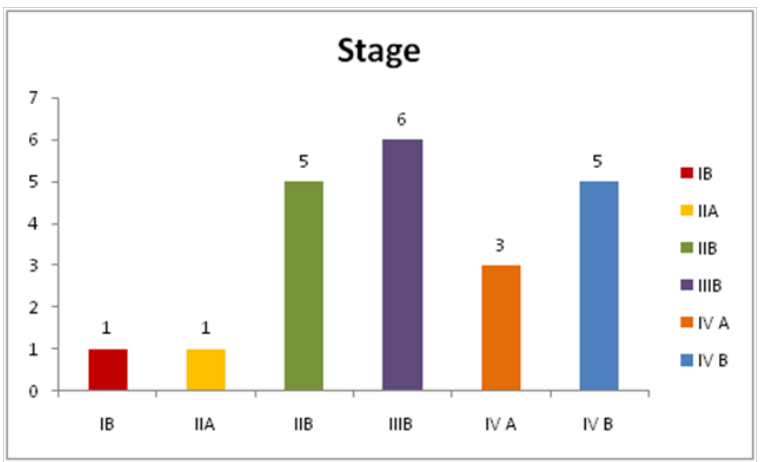

Figure 2 Stage distribution drawn for all the 21 participants. Most of the patients were IIB, IIB, and IVB stage.

\section{Conclusion}

Serum CEA has the potential of prognosticating CA cervix patients especially with the involvement of pelvic and paraaortic nodes. In our Study though small in size we found that it could be a very reliable marker for involvement of pelvic and paraaortic lymph nodes, where sometimes the CT or MRI could be of limited value if size of nodes were less than $1 \mathrm{~cm}$. The CEA values, both baseline and post treatment could be serve as a response indicator. This may be an invaluable tool for patient surveillance as detecting early failures may help to salvage the unfortunate patients and give them a chance to prolong their survival.

\section{Acknowledgements}

Author would like to express their special thanks and great appreciation to Mr. Nelson John for providing assistance in data analysis which helped to finish this project within the time frame.

\section{Conflicts of Interest}

The Author declared no potential conflict of interest with respect to the research authorship, and or publication of the article.

\section{Funding}

None.

\section{References}

1. Sturgeon CM, Duffy MJ, Stenman UH, et al. National Academy of Clinical Biochemistry laboratory medicine practice guidelines for use of tumor markers in testicular, prostate, colorectal, breast, and ovarian cancers. Clinical Chemistry. 2008;54(12):e11-e79.

2. Ueda Y, Enomoto T, Kimura T,et al. Serum biomarkers for early detection of gynecologic cancers. Cancers. 2008;2(2):1312-1327.

3. Sturgeon CM, Diamandis EP. Use of tumor markers in liver, bladder, cervical, and gastric cancers. Laboratory medicine practice guidelines. National Academy of Clinical Biochemistry, Washington, USA. 2010.

4. Sakuragi N, Satoh C, Takeda N, et al. Incidence and distribution pattern of pelvic and paraaortic lymph node metastasis in patients with stages IB, IIA, and IIB cervical carcinoma treated with radical hysterectomy. Cancer. 1999;85(7):1547-1554.

5. Gold MA, Tian C, Whitney CW, et al. Surgical versus radiographic determination of para-aortic lymph node metastases before chemoradiation for locally advanced cervical carcinoma. Cancer. 2008;112 (9):1954-1963.

6. Gouy S, Morice P, Narducci F, et al. Prospective multicenter study evaluating the survival of patients with locally advanced cervical cancer undergoing laparoscopic para-aortic lymphadenectomy before chemoradiotherapy in the era of positron emission tomography imaging. J Clin Oncol. 2013;31(24):3026-3033.

7. Frumovitz M, Querleu D, Gil-Moreno A, et al. Lymphadenectomy in Locally Advanced Cervical Cancer Study (LiLACS): phase iii clinical trial comparing surgical with radiologic staging in patients with stages IB2-IVA cervical cancer. J Minim Invasive Gynecol. 2014;21(1):3-8.

8. National Comprehensive Cancer Network. NCCN Clinical Practice Guidelines in Oncology: Cervical. Cancer. 2016.

9. Tan E, Gouvas N, Nicholls RJ, et al. Diagnostic precision of carcinoembryonic antigen in the detection of recurrence of colorectal cancer. Surgical Oncology. 2009;18(1):15-24.

10. Lo Gerfo P, Krupey J, Hansen HJ. Demonstration of an antigen common to several varieties of neoplasia:Assay using zirconyl phosphate gel. $N$ Eng J Med. 1971;285(3):138-141.

11. Kjorstad KE, Ørjasaester $\mathrm{H}$. The prognostic value of CEA determinations in the plasma of patients with squamous cell cancer of the cervix. Cancer. 1982;50(2):283-287.

12. Kjorstad KE, Ørjasaeter $H$. The prognostic significance of carcinoembryonic antigen determinations in patients with adenocarcinoma of the cervix. Gynecol oncol. 1984;19(3):284-289.

13. Huang EY, Huang YJ, Chanchien CC, et al. Pretreatment carcinoembryonic antigen level is a risk factor for para-aortic lymph node recurrence in addition to squamous cell carcinoma antigen following definitive concurrent chemoradiotherapy for squamous cell carcinoma of the uterine cervix. Radiat Oncol. 2012;7(1):1.

14. Meier W, Eiermann W, Stieber P, et al. Squamous cell carcinoma antigen and carcinoembryonic antigen levels as prognostic factors for the response of cervical carcinoma to chemotherapy. Gynecol Oncol. 1990;38(1):6-11.

15. Disaia PJ, Morrow CP, Haverback BJ, et al. Carcinoembryonic antigen in cancer of the female reproductive system: Serial plasma values correlated with disease state. Cancer. 1977;9(6):2365-2370.

16. Manetta A, Delgado G, Petrilli E, et al. The significance of paraaortic node status in carcinoma of the cervix and endometrium. Gynecol Oncol. 1986;23(3):284-290. 